\title{
A simplified route to the synthesis of CMK-3 replica based on precipitation polycondensation of furfuryl alcohol in SBA-15 pore system
}

Paula Niebrzydowska $^{\mathrm{a}}$, Rafał Janus ${ }^{\mathrm{a}}$, Piotr Kuśtrowski ${ }^{\mathrm{a},{ }^{* 1}}$, Sebastian Jarczewski ${ }^{\mathrm{a}}$, Anna Wach ${ }^{\mathrm{a}}$, Ana M. Silvestre-Albero ${ }^{\mathrm{b}}$, Francisco Rodríguez-Reinoso ${ }^{\mathrm{b}}$

${ }^{a}$ Department of Chemical Technology, Faculty of Chemistry, Jagiellonian University, Ingardena 3, 30-060 Kraków, Poland

${ }^{b}$ Departamento de Química Inorgánica, Universidad de Alicante, Apartado 99, E-03080 Alicante, Spain

\begin{abstract}
A novel method of synthesis of mesoporous, polymer-derived CMK-3 carbon replica was proposed. Instead of a multi-stage, time-consuming and toxic solvent involving procedure, the direct, acid-catalyzed precipitation polycondensation of furfuryl alcohol to poly(furfuryl alcohol) (PFA), as the carbon precursor, in the pore system of SBA-15 silica was used. The optimal PFA/SBA-15 mass ratio resulting in the complete pore filling was found. The final carbon material was obtained by carbonization of the formed composite and subsequent removal of silica by treatment with HF. Low-temperature sorption of nitrogen, powder X-ray diffraction and transmission electron microscopy confirmed the formation of well-ordered, hexagonal carbon mesostructure. The produced CMK-3 exhibited the presence of oxygencontaining surface groups, recognized as mainly carbonyl and carboxyl species by X-ray photoelectron spectroscopy and temperature-programmed desorption. The presence of these

\footnotetext{
${ }^{*}$ Corresponding author. Tel: +48 12 6632006; Fax: +48 12 6340515. E-mail address: kustrows@chemia.uj.edu.pl (P. Kuśtrowski)
} 
groups caused the mesoporous carbon to be catalytically active in the oxidative dehydrogenation of ethylbenzene to styrene.

\section{Introduction}

In 1999, Ryoo et al. described the synthesis of highly ordered mesoporous carbon (CMK-1) belonging to a group of novel materials called CMK-n (Carbon Mesostructured by KAIST) [1,2]. This first successful preparation of carbon negative of ordered mesoporous silica was inspiration for scientists to do advanced research on the development of carbon mesostructures exhibiting some valuable advantages, i.e. well ordered, adjustable porosity, large pore size and volume as well as high specific surface area [3]. Many different silica matrices (so called hard templates) and carbon precursors were used to produce carbon replicas. Generally, their synthesis included four essential steps: (i) preparation of the hard template, (ii) deposition of the carbon source in the channels of ordered mesoporous silica by e.g. wetness impregnation or chemical vapor deposition (CVD), (iii) carbonization of the carbon precursor/silica composite under an inert atmosphere, and (iv) removal of the silica template using hydrofluoric acid or alkali solution. It was found that improved textural and structural parameters (e.g. mesoscopic regularity, surface area, pore volumes, graphitization degree and concentration of functional surface groups) of the final carbon mesostructures were achieved for the replicas based on polymer-derived carbons [4].

The synthesis of CMK-3 replica was first proposed by Jun et al., who used SBA-15 mesoporous silica as the hard template and sucrose as the source of carbon [5]. The CMK-3 carbon is an inverse replica of mesoporous silica with p6mm space group, in which the 2D hexagonally arranged carbon rods are interconnected by the carbon bridges. Many different carbon precursors, such as sucrose, furfuryl alcohol (FA), naphthalene, anthracene, aniline, propylene, acrylonitrile and phenolic resin, were tested in the formation of CMK-3 replica [4,6-10]. Among them, 
poly(furfuryl alcohol) (PFA) was recognized as a very promising carbon precursor. The 3-D thermosetting network of PFA exhibits high resistance to loss of carbon atoms under the carbonization conditions hence it transforms to carbon with a high yield and forms a rigid skeleton of carbon replica [4]. In the case of PFA-derived CMK-3 replica synthesis, the SBA-15 hard template was modified with the carbon precursor by the introduction of furfuryl alcohol (FA) into the pore system of silica initially pre-treated with acidic catalyst of polycondensation (e.g. $\mathrm{AlCl}_{3}$, oxalic acid). Fuertes et al. [11-13] synthesized CMK-3 using the deposition of FA by chemical vapor deposition or incipient wetness impregnation on SBA-15 modified with ptoluenesulfonic acid. The CMK-3 synthesis method based on esterification of silanol groups with FA was also shown by Yokoi et al. [6]. In this approach, the outgassed silica template was introduced into the toluene solution of FA and refluxed for $24 \mathrm{~h}$ in order to remove produced $\mathrm{H}_{2} \mathrm{O}$ from a water-toluene azeotropic system. The esterification method significantly improved the homogeneity of PFA dispersion on the SBA-15 surface.

The above mentioned methods of formation of carbon/SBA-15 composites based on the FA carbon precursor are multi-stage and time-consuming. In addition, they often involve toxic solvents (for example aromatic hydrocarbons). In this work we describe a new approach to the synthesis of CMK-3 replica based on the FA precursor. Instead of the complicated route of PFA formation, we propose the simple precipitation of PFA formed by the FA polycondensation in water slurry of pristine SBA-15, which resulted in homogeneous distribution of the formed polymer in the channels of silica template. Finally, we obtained the CMK-3 material composed of carbon nanorods surrounded by a carbon shell formed by carbonization of PFA deposited on the outer surface of SBA-15 particles, which can improve the stability of carbon mesostructure. 


\section{Experimental}

\subsection{Synthesis of CMK-3 carbon replica}

SBA-15 was synthesized according the procedure presented earlier by Michorczyk et al. [14] A molar gel composition: 1.00 tetraethyl orthosilicate (TEOS): 0.02 Pluronic P123: $2.89 \mathrm{HCl}$ : 117.92 $\mathrm{H}_{2} \mathrm{O}$ was used. Briefly, $8.0 \mathrm{~g}$ of Pluronic P123 $\left(\mathrm{EO}_{20} \mathrm{PO}_{70} \mathrm{EO}_{20}\right.$, Aldrich) was stirred (1000 rpm) in a mixture containing $60.0 \mathrm{~g}$ of distilled water and $120.0 \mathrm{~g}$ of $2 \mathrm{M} \mathrm{HCl}$ at $35^{\circ} \mathrm{C}$ until dissolved. Afterwards, 17.0 g of TEOS (99.0\%, Aldrich) as a silica source was added dropwise (2 drops/s) to the prepared solution. The mixture was stirred (400 rpm) at $35^{\circ} \mathrm{C}$ for $20 \mathrm{~h}$ and then aged at $90^{\circ} \mathrm{C}$ for $24 \mathrm{~h}$. The formed precipitate was filtered, washed with distilled water and dried at $60^{\circ} \mathrm{C}$ overnight. Finally, in order to remove the polymeric template, the material was calcined under air atmosphere at $550^{\circ} \mathrm{C}$ with a heating rate of $1^{\circ} \mathrm{C} / \mathrm{min}$ and an isothermal period of $10 \mathrm{~h}$.

The obtained SBA-15 sample was used for the synthesis of CMK-3 materials. Poly(furfuryl alcohol) was deposited in the channel system of SBA-15 by the precipitation polycondensation of FA (98\%, Aldrich) in an aqueous slurry of silica template containing $\mathrm{HCl}$ (33\%, Polish Chemical Reagents). The intended PFA/SBA-15 mass ratios of 1.00-2.50 (with a step of 0.25) were adjusted by using an appropriate FA volume. The HCl/FA molar ratio was kept constant at 6:1. An amount of $3.0 \mathrm{~g}$ of freshly calcined silica matrix was introduced into a round-bottom flask (250 $\mathrm{cm}^{3}$ ) equipped with a reflux condenser and placed on a magnetic stirrer. Then, adequate volumes of distilled water and FA were added to obtain a total mixture volume of $100 \mathrm{~cm}^{3}$. The slurry was stirred for $0.5 \mathrm{~h}$ at room temperature. Subsequently, the proper volume of $\mathrm{HCl}$ was added, the mixture was heated up to $100^{\circ} \mathrm{C}$ and stirred at this temperature for 6 h. The obtained brown solid was filtered, washed with distilled water and dried overnight at room temperature. Subsequently, the PFA/silica composites were carbonized in a tubular furnace under a flow of inert gas $\left(\mathrm{N}_{2}, 40\right.$ $\mathrm{cm}^{3} / \mathrm{min}$ ) at $850^{\circ} \mathrm{C}$ with a heating rate of $1^{\circ} \mathrm{C} / \mathrm{min}$ and an isothermal period of $4 \mathrm{~h}$. Finally, the 
silica hard template was removed by two times treatment in a 5\% hydrofluoric acid solution at room temperature. The CMK-3 carbon was filtered, washed with distilled water and ethyl alcohol (96\%, Aldrich) and dried at $40^{\circ} \mathrm{C}$ overnight. The carbonized samples were labeled as PFAx/SBA15 (where $x$ is the intended $\mathrm{PFA} / \mathrm{SiO}_{2}$ mass ratio), and the final replicas were marked as CMK-3 $(x)$.

\subsection{Characterization methods}

Nitrogen adsorption-desorption isotherms were collected at $-196^{\circ} \mathrm{C}$ in a home-made fully automated equipment, designed and constructed by the Advanced Materials group (LMA), and commercialized as N2Gsorb-6 (Gas to Materials Technologies). Before the measurements, the samples were outgassed at $250^{\circ} \mathrm{C}$ for $4 \mathrm{~h}$ at a base pressure of $1.3 \cdot 10^{-3} \mathrm{~Pa}$. The specific surface areas were calculated using the Brunauer-Emmett-Teller (BET) equation. The pore size distribution of SBA-15 matrix was determined basing on the equilibrium model of non-local density functional theory (NLDFT) [15] dedicated for cylindrical pores of siliceous adsorbents, whereas the equilibrium model of quenched solid density functional theory (QSDFT) [16] was used to determine the pore size distribution of CMK-3 replica. The same models were applied to calculate micro- and mesopore volumes. The analyses were performed in the ASiQwin software (version 1.11) by Quantachrome.

The structure of the obtained samples was examined by X-ray powder diffraction (XRD) using a Bruker D2 Phaser instrument with a LYNXEYE detector. The diffraction patterns were collected using CuK $\alpha$ radiation $(\lambda=1.54184 \AA)$ in a $2 \theta$ range of $0.75 \div 3.00^{\circ}$ with a step of $0.02^{\circ}$.

Transmission electron microscopy (TEM) imaging was studied with a JOEL microscope (model JEM-2010) equipped with an INCA Energy TEM 100 analytical system and a SIS MegaView II 
camera, working at $200 \mathrm{kV}$. Prior to the experiments, the samples were suspended in ethanol and placed on copper grids with a carbon film support (LASEY).

TG measurements were carried out with a SDT Q600 (TA Instruments) in air atmosphere (a flow rate of $100 \mathrm{~cm}^{3} / \mathrm{min}$ ) from $30^{\circ} \mathrm{C}$ to $1000^{\circ} \mathrm{C}$ at a heating rate of $20^{\circ} \mathrm{C} / \mathrm{min}$.

X-ray photoelectron spectroscopy (XPS) measurements were performed with a Prevac photoelectron spectrometer equipped with a hemispherical analyzer VG SCIENTA R3000. The spectra were taken using a monochromatized aluminum source $\mathrm{AlK} \alpha(\mathrm{E}=1486.6 \mathrm{eV})$. The base

pressure in the analytical chamber was $5 \cdot 10^{-9}$ mbar. The binding energy scale was calibrated using the $\mathrm{Au} 4 \mathrm{f}_{7 / 2}$ line of a cleaned gold sample at $84.0 \mathrm{eV}$. The surface composition of CMK-3 material was studied based on the areas and binding energies of $\mathrm{C} 1 \mathrm{~s}$ and $\mathrm{O}$ 1s core levels. The spectra were fitted using the CasaXPS software.

Temperature-programmed desorption (TPD) experiment was carried out to evaluate the amount and nature of the oxygen-containing surface groups present on the surface of the fresh PFAderived carbon replica. $100 \mathrm{mg}$ of sample was placed in a U-shaped quartz reactor directly connected to a quadrupole mass spectrometer (Balzer MSC 200) and heated from $20^{\circ} \mathrm{C}$ to $1000^{\circ} \mathrm{C}$ under a helium flow $\left(50 \mathrm{~cm}^{3} / \mathrm{min}\right)$ and a heating rate of $10^{\circ} \mathrm{C} / \mathrm{min}$. The amounts of evolved CO and $\mathrm{CO}_{2}$ were calculated quantitatively after calibration based on calcium oxalate $\left(\mathrm{CaC}_{2} \mathrm{O}_{4} \cdot \mathrm{H}_{2} \mathrm{O}\right)$ decomposition [17].

\subsection{Catalytic tests}

The synthesized PFA-derived carbon material was tested as a catalyst in the oxidative dehydrogenation of ethylbenzene (EB) to styrene in the presence of oxygen at the molar ratio $\mathrm{EB}: \mathrm{O}_{2}=1: 1$. The catalytic runs were carried out in a flow-type quartz microreactor filled with 50 mg of catalyst placed in the central position of the reactor onto a quartz wool plug. The flow of 
the gaseous reactants was controlled by mass flow controllers (Brooks 4800 Series). The total flow was $3 \mathrm{dm}^{3} / \mathrm{h}$, which contained $0.024 \mathrm{dm}^{3}$ of $\mathrm{O}_{2} / \mathrm{h}$ diluted in helium. EB was fed continuously into the system by saturation of the $\mathrm{He}+\mathrm{O}_{2}$ mixture in a saturator filled with liquid EB kept at $25^{\circ} \mathrm{C}$. The analysis of reaction products was performed using a gas chromatograph Bruker 450-GC equipped with three packed columns (Porapak Q, Molecular Sieve 5A, and Chromosorb W-HP) and three detectors (two flame ionization detectors - including one coupled with catalytic methanizer - and thermal conductivity detector). Prior to the catalytic run, the sample was outgassed at $200^{\circ} \mathrm{C}$ for $30 \mathrm{~min}$ in a flow of helium $\left(3 \mathrm{dm}^{3} / \mathrm{h}\right)$. Then, the temperature was increased to the reaction temperature (300, 350, 400, 450 and $500^{\circ} \mathrm{C}$ ) and dosing of $\mathrm{EB}$ and the $\mathrm{He}+\mathrm{O}_{2}$ mixture started. The first GC analysis was carried out after 15 min. Further analyses were collected at 40 min time intervals during the total reaction time of $7 \mathrm{~h}$. Conversion of EB, yield of styrene and selectivity to reaction products were calculated according to the following equations:

$$
\begin{gathered}
C_{E B}=\frac{F_{E B, 0}-F_{E B}}{F_{E B, 0}} \cdot 100 \% \\
Y_{i}=\frac{F_{i}}{F_{E B, 0}} \cdot 100 \% \\
S_{i}=\frac{Y_{i}}{C_{E B}} \cdot 100 \%
\end{gathered}
$$

where: $C_{E B}$ - conversion of ethylbenzene; $F_{E B, 0}$ and $F_{E B}-$ molar flow rate of EB in the inlet and outlet streams; $Y_{i}$ - yield of $i$ product; $F_{i}$ - molar flow rate of EB transformed into $i$ product; $S_{i}$ selectivity of $i$ product. 


\section{Results and discussion}

\subsection{Introduction of different amounts of PFA into the channels of SBA-15}

The low-temperature nitrogen adsorption isotherms collected for the carbonized PFA/SBA-15 composites in comparison to the isotherm of pristine SBA-15, which is of type IV with a H1 hysteresis loop $[9-10,18]$, are displayed in Fig. 1. As seen, the capillary condensation in the case of SBA-15 occurs at $p / p_{0}$ between 0.6 and 0.7 , while the carbonized PFA/SBA-15 composites display the condensation step in the $p / p_{0}$ range of $0.5-0.7$. Moreover, the hysteresis loop for the carbonizates narrows with increasing content of carbonized polymer. These facts prove that the formed polymeric species were successfully deposited in the silica mesochannels. The covering of the inner surface of the SBA-15 template with increasing amounts of PFA results in a progressive blocking of mesopores. For the samples with the intended PFA/SBA-15 mass ratio of 2.00 and higher the hysteresis loop disappears completely suggesting complete filling of the mesopore system of silica matrix.

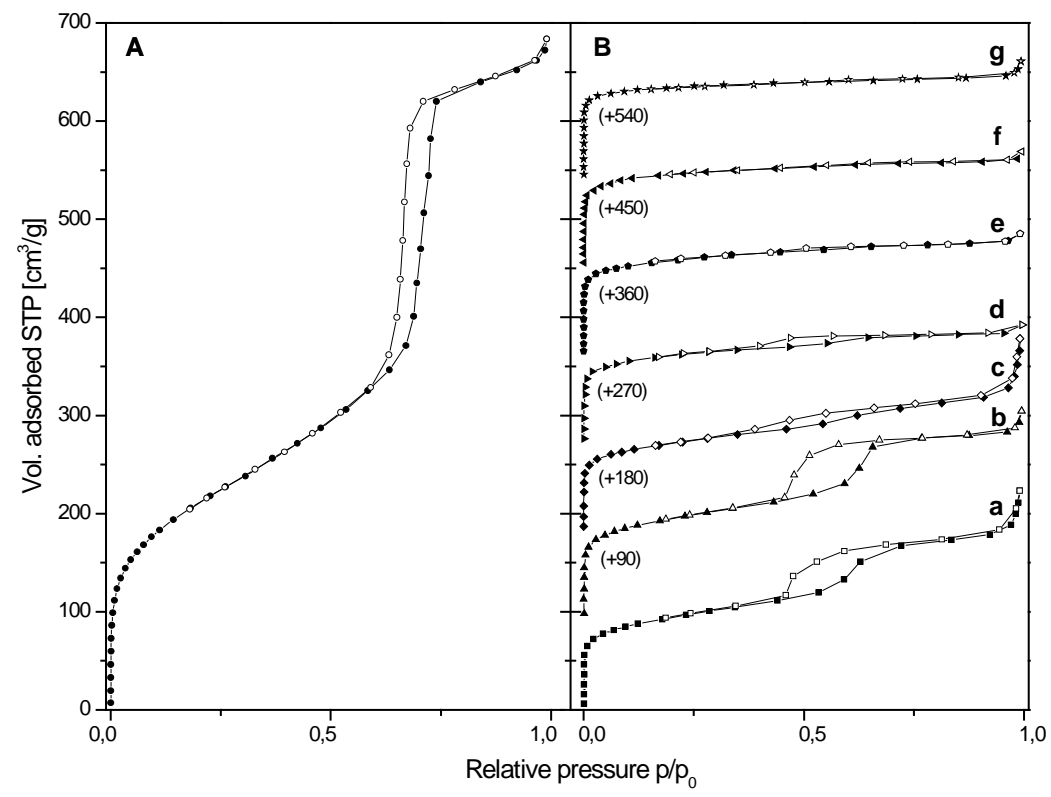

Fig. $1-\mathrm{N}_{2}$ isotherms collected for neat SBA-15 silica (A) and PFA/SBA-15 carbonizates (B): PFA1.00/SBA-15 (a), PFA1.25/SBA-15 (b), PFA1.50/SBA-15 (c), PFA1.75/SBA-15 (d), 
PFA2.00/SBA-15 (e), PFA2.25/SBA-15 (f), PFA2.50/SBA-15 (g). For clarity the isotherms of PFA1.25 $\div 2.50 /$ SBA-15 were shifted vertically by $90,180,270,360,450$ and $540 \mathrm{~cm}^{3} / \mathrm{g} \mathrm{STP}$, respectively.

Table 1 - Amounts of PFA introduced to SBA-15 by precipitation polycondensation and textural parameters of the resulting PFA/SBA-15 carbonizates.

\begin{tabular}{|c|c|c|c|c|}
\hline \multirow{2}{*}{$\begin{array}{l}\text { Intended mass } \\
\text { ratio of } \\
\text { PFA/SBA-15 }\end{array}$} & \multirow{2}{*}{$\begin{array}{c}\text { Content of PFA } \\
\text { introduced } \\
\left.\text { [g } \mathrm{g}_{\mathrm{PFA}} / \mathrm{g}_{\text {composite }}\right]\end{array}$} & \multicolumn{3}{|c|}{ Textural parameters of carbonized composites } \\
\hline & & $\mathrm{S}_{\mathrm{BET}}\left[\mathrm{m}^{2} / \mathrm{g}\right]$ & $\mathrm{V}_{\text {micro }}\left[\mathrm{cm}^{3} / \mathrm{g}\right]$ & $\mathrm{V}_{\text {meso }}\left[\mathrm{cm}^{3} / \mathrm{g}\right]$ \\
\hline 0.00 & - & 990 & 0.24 & 0.75 \\
\hline 1.00 & 0.45 & 324 & 0.14 & 0.14 \\
\hline 1.25 & 0.53 & 346 & 0.16 & 0.13 \\
\hline 1.50 & 0.56 & 311 & 0.14 & 0.09 \\
\hline 1.75 & 0.61 & 310 & 0.15 & 0.03 \\
\hline 2.00 & 0.65 & 332 & 0.15 & 0.02 \\
\hline 2.25 & 0.63 & 318 & 0.14 & 0.02 \\
\hline 2.50 & 0.66 & 302 & 0.14 & 0.02 \\
\hline
\end{tabular}

Taking into account the textural parameters of the PFA/SBA-15 carbonizates, presented in Table 1, we conclude that deposited PFA influences significantly the textural parameters of a formed composite. The introduction of polymer into the mesochannels is confirmed by a simultaneous decrease in the BET surface area $\left(\mathrm{S}_{\mathrm{BET}}\right)$ and the mesopore volume $\left(\mathrm{V}_{\text {meso }}\right)$. On the other hand, regardless of the decrease in mesoporosity with rising PFA content, the volume of micropores $\left(\mathrm{V}_{\text {micro }}\right)$ of all the studied carbon/silica composites remains constant at $0.14-0.16$ $\mathrm{cm}^{3} / \mathrm{g}$. We attribute this effect to blocking with the SBA-15 pore system (both micro- and mesopores) and a subsequent development of micropore volume formed inside the carbon material as well as between carbon and silica walls during the carbonization step. Shrinkage of 
PFA-derived carbon deposited in the silica channels after thermal treatment at high temperatures was observed previously by other authors [19-21].

Table 1 also presents the real poly(furfuryl alcohol) contents in the PFA/SBA-15 precursors determined by TG. It can be found that the conditions of polycondensation process allowed obtaining a relatively high conversion of FA to PFA in the range of intended PFA/SBA-15 mass ratios between 1.00 and 2.00. However, in the case of the higher concentrations of FA in the reaction slurry the presence of brown water soluble oligomeric structures in the filtrate was observed. That suggests a lower effectiveness of deposition of polymer species at a high FA content, which was confirmed by the TG analysis (Fig. 2).

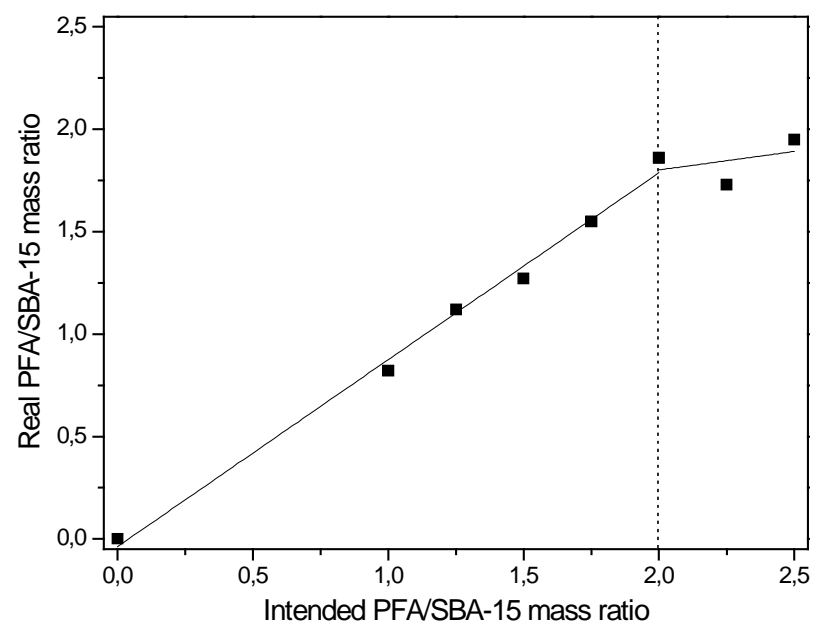

Fig. 2 - Effectiveness of poly(furfuryl alcohol) accumulation in silica matrix pore system.

It should be noticed that the most effective incorporation of polymer into the prepared composites occurred until the complete filling of the silica template mesochannels. This deposition degree is found for the PFA2.00/SBA-15 sample. A further increase in the amount of formed PFA led to its weak interaction with the support surface. In the volume of liquid phase mainly PFA oligomers 
were produced, which were easily lost during the separation of solid after the finished modification.

\subsection{Changes in structure and morphology of the PFA2.00/SBA-15 sample at different stages of synthesis}

The results of low-temperature adsorption of $\mathrm{N}_{2}$ and thermal analysis indicated the PFA2.00/SBA-15 composite as the most suitable precursor to obtain structurally ordered and stable carbon replica. Therefore, we selected this material for investigation of structural transformations from the silica precursor to the final CMK-3 carbon replica.

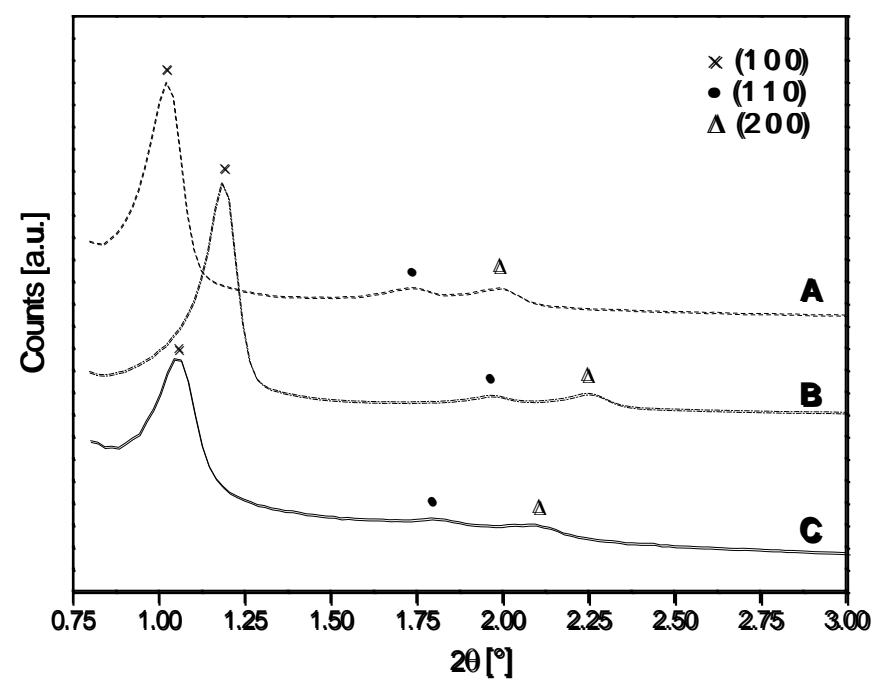

Fig. 3 - Low-angle X-ray powder diffraction patterns for pristine SBA-15 (A), SBA-15 calcined at $850^{\circ} \mathrm{C}(\mathrm{B})$ and corresponding $\mathrm{CMK}-3$ (2.00) replica (C).

The XRD pattern for the SBA-15 support (Fig. 3, A) features three distinct peaks at $2 \theta$ angles of $0.98^{\circ}, 1.67^{\circ}$, and $1.93^{\circ}$, which can be indexed as (1 00 ), $\left(\begin{array}{lll}1 & 1 & 0\end{array}\right)$, and $\left(\begin{array}{lll}2 & 0 & 0\end{array}\right)$ reflections of a twodimensional well ordered hexagonal mesostructure (the space group of p6mm) [22-25]. The 
determined $\mathrm{d}_{100}$ interplanar spacing equal to $9.0 \mathrm{~nm}$ corresponds to the value of lattice parameter a of $10.4 \mathrm{~nm}$, which was calculated according to the equation:

$$
a=\frac{2 d_{100}}{\sqrt{3}}
$$

and is considered as the distance between the centers of adjacent pores.

In case of the CMK-3 replica the XRD data (Fig. 3, C) indicate that the hexagonal arrangement of the channels with the space group of $\mathrm{p} 6 \mathrm{~mm}$ is preserved. The $\mathrm{d}_{100}$ spacing value for CMK$3(2.00)$ is $8.3 \mathrm{~nm}$, while the lattice parameter a is equal to $9.7 \mathrm{~nm}$.

The $\mathrm{d}_{100}$ spacing value for pristine SBA-15 calcined at the same conditions as the PFA2.00/SBA15 composite $\left(850^{\circ} \mathrm{C}, 4 \mathrm{~h}\right)$ is equal to $7.4 \mathrm{~nm}$ with the value of lattice parameter a of $8.6 \mathrm{~nm}$ (Fig. 3, B). As seen, both parameters are about $1 \mathrm{~nm}$ lower compared with the CMK-3 (2.00) replica. It should be therefore concluded that the polymer filled mesochannels of SBA-15 exhibit clearly higher resistance to shrinkage of the structure during the high temperature calcination.

The TEM images displayed in Fig. 4 exhibit large domains of the neat silica matrix (A), the PFA/SBA-15 composites before and after carbonization $(B, C)$ and the resultant replica (D). These micrographs clearly confirm that the structural arrangement characteristic for SBA-15 is preserved in all synthesis steps. It can be seen that SBA-15 has a very uniform distribution of hexagonal channels and CMK-3 is exactly an inverse structure of the pristine SBA-15 matrix. The carbon nanorods forming the CMK-3 structure are interconnected by spacers, which derived from the carbonaceous species that filled the micropores present within the SBA-15 walls. Furthermore, we noticed that the formed CMK-3 particles are enveloped by a thin carbon shell originated from carbonized PFA deposited on the outer surface of silica matrix (Fig. 4, D and D'). 

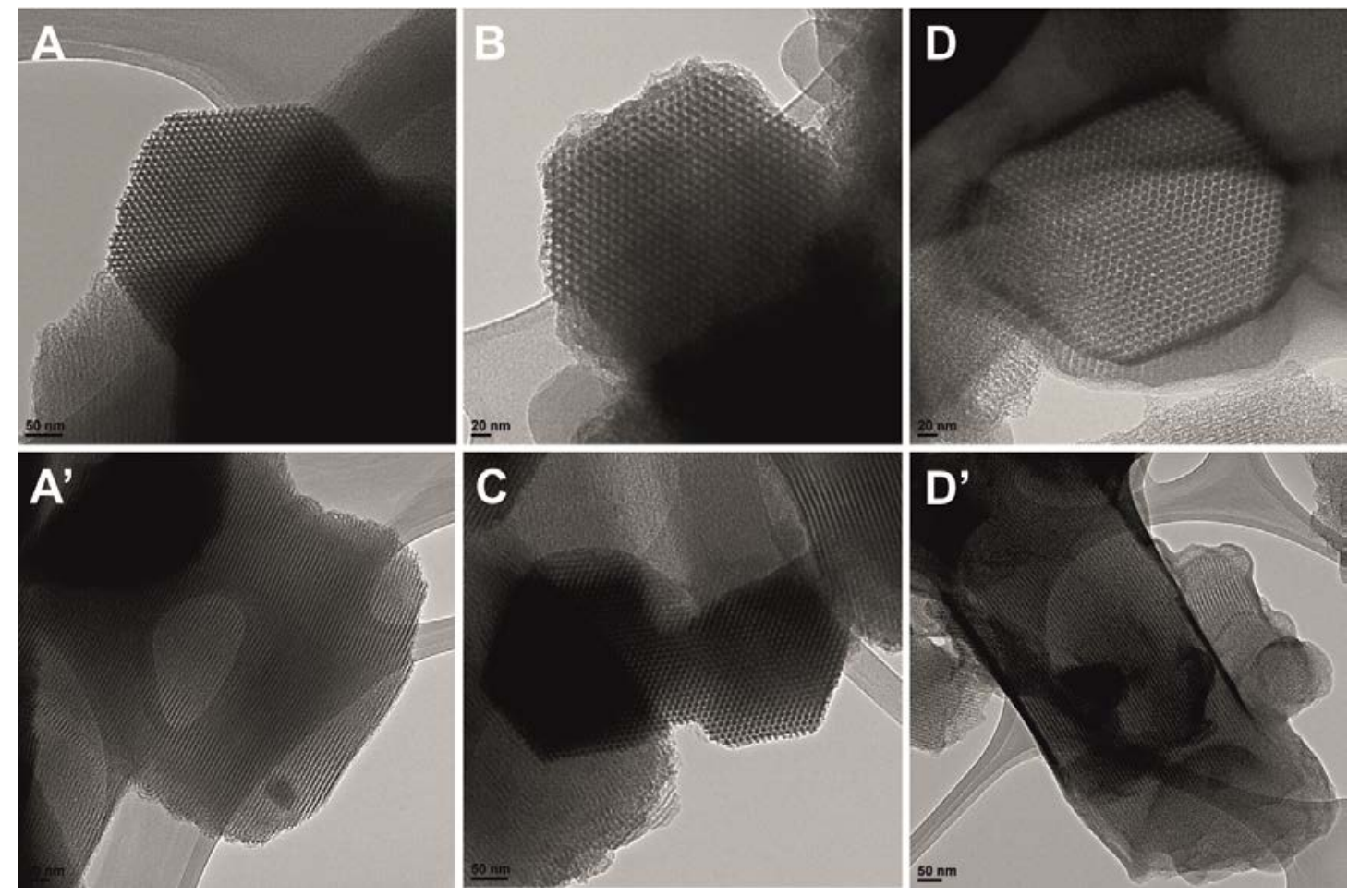

Fig. 4 - TEM images for SBA-15 taken perpendicular (A) and parallel (A') to the pores, PFA(2.00) deposited in the SBA-15 channels (B), PFA2.00/SBA-15 composite after carbonization (C) and corresponding CMK-3 (2.00) replica (D and D').

\subsection{Textural parameters of CMK-3 (2.00) replica}

The nitrogen adsorption-desorption isotherm recorded for the CMK-3 (2.00) replica, shown in Fig. 5, is of type IV with a well-defined capillary condensation stage at relative pressures $p / p_{0}$ between of 0.4 and 0.7 , indicating very uniform mesoporosity [26,27]. The pore size distribution curve calculated basing on the equilibrium QSDFT model reveals two maxima at 0.9 and $3.1 \mathrm{~nm}$. The bimodal porosity of the synthesized carbon material can be attributed to the presence of mesopores formed between the carbon rods after the removal of silica matrix as well as to micropores generated inside the carbon rods. The presence of such microporosity is typical of carbonized PFA [28,29]. 


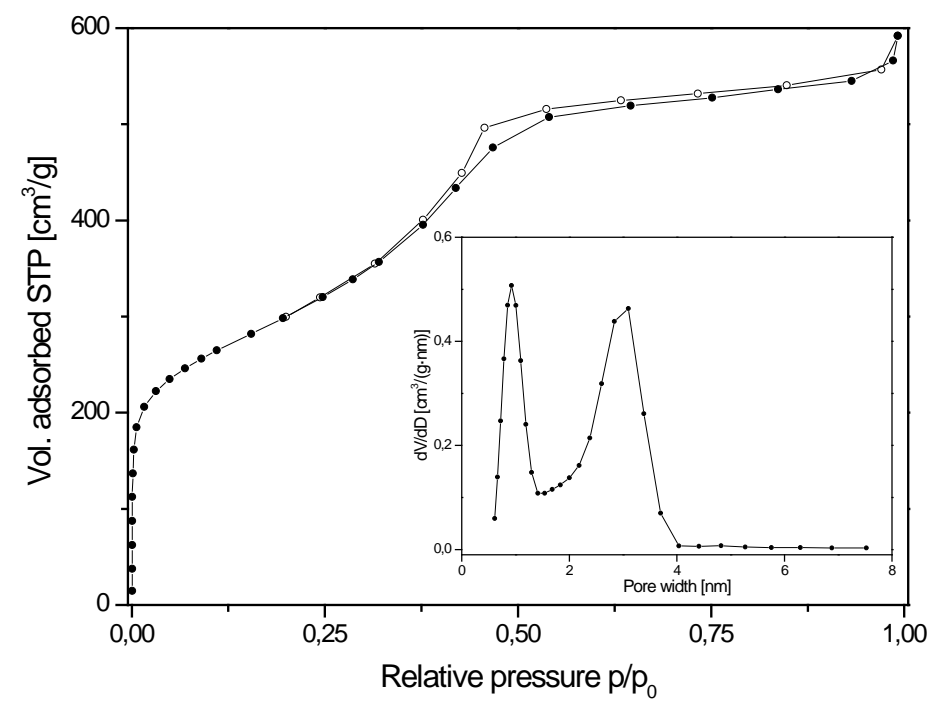

Fig. 5 - Low-temperature $\mathrm{N}_{2}$ adsorption-desorption isotherm and pore size distribution (inset; QSDFT model) of the CMK-3 (2.00) replica.

The collected isotherm allowed the calculation of textural parameters of the CMK-3 replica. The obtained material shows the high BET surface area of $1067 \mathrm{~m}^{2} / \mathrm{g}$, and the volume of mesopore and micropore equal to 0.52 and $0.31 \mathrm{~cm}^{3} / \mathrm{g}$, respectively. These values are comparable to data published previously for CMK-3 materials [10,22,24]. Moreover, carbon nanorods diameter $\left(d_{r}\right)$ can be estimated using the geometrical considerations proposed by Kruk et al. [30,31] (cf. Fig. S1 and Table S1 in Supplementary Data). Assuming that the distance between adjacent carbon rods is equal to the thickness of SBA-15 walls $\left(\mathrm{b}_{\mathrm{d}}\right)$, the $d_{r}$ value of $8.3 \mathrm{~nm}$ for CMK-3 (2.00) can be calculated using the following formula:

$$
d_{r}=a_{C M K-3}-b_{d}
$$

where $\mathrm{a}_{\mathrm{CMK}-3}$ is a lattice parameter of CMK-3.

We also removed the silica template from the carbonized PFA/SBA-15 composites with incompletely filled pore system (the materials with the intended PFA/SBA-15 mass ratios below 2.00), and the carbon materials with bimodal mesoporosity were obtained. The carbon replica 
derived from the PFA1.25/SBA-15 composite shows $\mathrm{N}_{2}$ adsorption isotherm (cf. Fig. S2 in Supplementary Data) with two steps corresponding to capillary condensation in mesopores. Beside the channels with diameter typical of space between carbon nanorods, wider mesopores exist, which can be assigned to carbon nanotubes like in a CMK-5 replica.

\subsection{Surface composition of CMK-3 (2.00) replica}

TPD and XPS measurements were carried out in order to determine the amount and type of oxygen-containing groups present on the fresh CMK-3 (2.00) surface.

The desorption profiles of $\mathrm{CO}$ and $\mathrm{CO}_{2}$ measured within the temperature range of $25-1000^{\circ} \mathrm{C}$ are shown in Fig. 6, whereas Table 2 includes the total concentration of oxygen-containing surface groups, which decompose forming $\mathrm{CO}$ and $\mathrm{CO}_{2}$. It is assumed that the type of evolved gaseous products and the peak temperatures evidence the presence of specific surface functionalities [32].

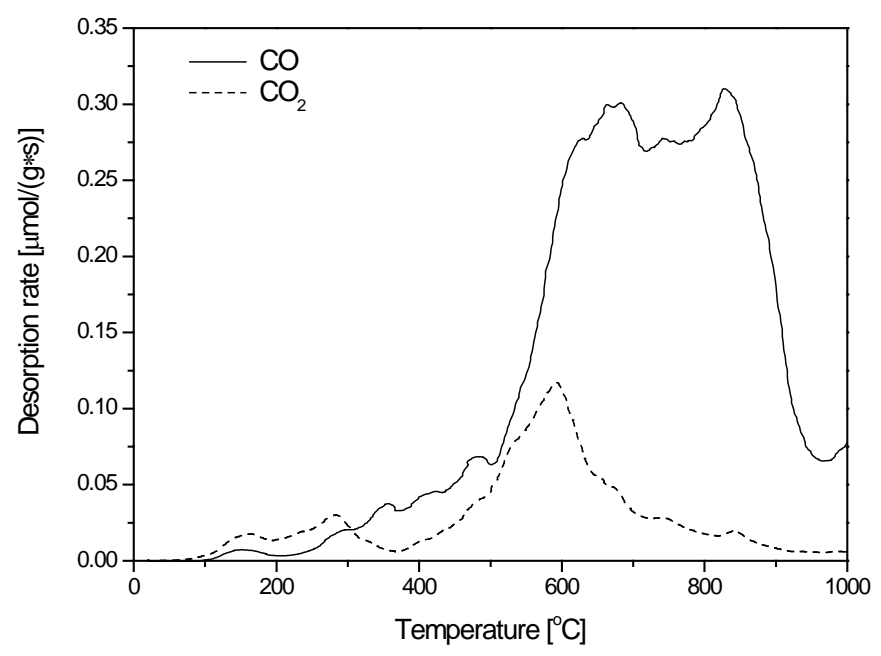

Fig. 6 - Temperature-programmed desorption profiles of $\mathrm{CO}$ and $\mathrm{CO}_{2}$ for the PFA-derived CMK3 (2.00) replica. 
In the case of the studied replica different forms of surface entities can be identified. The desorption of $\mathrm{CO}_{2}$ at temperatures below $350^{\circ} \mathrm{C}$ is assigned to decomposition of carboxyl groups. However, the intense $\mathrm{CO}_{2}$ evolution observed with the maximal rate at $590^{\circ} \mathrm{C}$ reveals that a majority of $\mathrm{COO}^{-}$species exists in the form of carboxylic anhydrides and lactones. On the other hand, two distinct maxima observed in the CO-TPD profile at about $650^{\circ} \mathrm{C}$ and $820^{\circ} \mathrm{C}$ correspond to decomposition of phenols, ethers, and carbonyl groups [17,31]. Generally, it should be underscored that the surface of CMK-3 (2.00) material contains very stable functional groups. It suggests that this replica can be used even in high-temperature applications (e.g. in catalysis).

Table 2 - Amounts of $\mathrm{CO}_{2}$ and $\mathrm{CO}$ evolved during the TPD experiments for the PFA-derived CMK-3 (2.00) replica.

\begin{tabular}{cccc}
\hline \multirow{2}{*}{ Sample } & $\mathrm{CO}$ & $\mathrm{CO}_{2}$ & \\
\cline { 2 - 3 } & {$[\mathrm{mmol} / \mathrm{g}]$} & {$[\mathrm{mmol} / \mathrm{g}]$} & \\
\hline $\mathrm{CMK}-3(2.00)$ & 0.71 & 0.16 & 4.4 \\
\hline
\end{tabular}

From the TPD experiments the total amounts of functional groups distributed on the whole surface (external and internal) were determined. Nevertheless, the content of these species on the external surface, which is the most accessible for the molecules from the gas phase, is interesting from the catalytic point of view. Therefore, the XPS measurement was performed for information on the elemental composition of the outermost few atomic layers of replica external surface.

The O 1s and C 1s spectra of CMK-3 (2.00) are illustrated in Fig. S3 (Supplementary Data), and the contribution of particular O- and C-containing species is summarized in Table 3. The O 1s core level spectrum was fitted with four peaks, indicating the presence of: (i) carbonyl groups $(\mathrm{C}=\mathrm{O})$ with binding energy of $530.8 \mathrm{eV}$, (ii) hydroxyl groups $(\mathrm{C}-\mathrm{OH})$ and oxygen atoms doubly 
bonded to carbon atoms in carboxyl groups ( $\mathrm{COOH})$ with binding energy of $533.0 \mathrm{eV}$, (iii) oxygen atoms single bonded to carbon atoms in carboxyl groups $(\mathrm{CO} \underline{\mathrm{OH}})$ with binding energy of $534.4 \mathrm{eV}$ and (iv) adsorbed water $(536.6 \mathrm{eV})[34,35]$. The presence of these groups was also confirmed by the C 1s core level spectrum, which was deconvoluted into four individual peaks. The predominant, asymmetric peak with an exponential tail, centered at $284.3 \mathrm{eV}$, was attributed to carbon atoms in graphitic and disordered carbon species $\left(\mathrm{C}=\mathrm{C} \mathrm{sp}^{2}\right.$ and $\left.\mathrm{C}-\mathrm{C} \mathrm{sp}^{3}\right)$. The shape of this peak is typical for carbonaceous materials with graphite-like structure [36]. The additional, symmetrical peaks centered at $286.3 \mathrm{eV}, 287.4 \mathrm{eV}$ and $288.4 \mathrm{eV}$ were assigned to $\mathrm{C}-\mathrm{OH}, \mathrm{C}=\mathrm{O}$ and $\mathrm{COOH}$ groups, respectively $[34,35,37]$. The polyaromatic character of carbon replica was furthermore confirmed by the presence of signal above $290.0 \mathrm{eV}$. This peak corresponds to shakeup satellite due to $\pi-\pi^{*}$ transitions in aromatic rings [34,36].

The $\mathrm{CO} / \mathrm{CO}_{2}$ molar ratio determined by XPS is significantly higher than that measured in TPD. It suggests that the concentration of carbonyl functionalities in relation to carboxyl groups is higher on the external surface of CMK-3 than on the internal one.

Table 3 - Concentration of carbon and oxygen species on the surface of fresh CMK-3 (2.00) replica and this material after catalytic runs at different temperatures determined by XPS.

\begin{tabular}{|c|c|c|c|c|c|c|c|c|}
\hline \multirow{3}{*}{ Sample } & \multicolumn{7}{|c|}{ Atomic concentration [\%] } & \multirow{3}{*}{$\mathrm{CO} / \mathrm{CO}_{2}$} \\
\hline & \multicolumn{4}{|c|}{ Carbon } & \multicolumn{3}{|c|}{ Oxygen } & \\
\hline & $\begin{array}{l}\underline{C}=\mathrm{C} \mathrm{sp}{ }^{2} \\
\underline{\mathrm{C}}-\mathrm{C} \mathrm{sp}{ }^{3}\end{array}$ & $\begin{array}{l}\underline{\mathrm{C}}- \\
\mathrm{OH}\end{array}$ & $\underline{\mathrm{C}}=\mathrm{O}$ & $\underline{\mathrm{COOH}}$ & $\mathrm{C}=\underline{\mathrm{O}}$ & $\begin{array}{c}\underline{\mathrm{OH}}, \\
\mathrm{C} \underline{\mathrm{OOH}}\end{array}$ & $\mathrm{CO} \underline{\mathrm{H}}$ & \\
\hline fresh & 92.40 & 2.29 & 1.15 & 0.18 & 1.14 & 2.47 & 0.18 & 6.4 \\
\hline 300 & 87.34 & 3.84 & 1.91 & 0.30 & 1.91 & 4.14 & 0.30 & 6.4 \\
\hline 400 & 87.64 & 3.40 & 2.41 & 0.21 & 2.41 & 3.60 & 0.21 & 11.5 \\
\hline 500 & 89.76 & 2.90 & 1.90 & 0.18 & 1.90 & 3.08 & 0.18 & 10.6 \\
\hline
\end{tabular}


The appearance of oxygen-containing groups on the surface of carbonized sample should be connected with the mechanism of decomposition of PFA supported on mesoporous silica and reoxidation of PFA-derived carbonizate. It has been shown that $\mathrm{SiO}_{2}$-deposited PFA undergoes structural transformation including some steps such as: opening the furan ring with the formation of $\gamma$-diketone moieties and subsequent deeper carbonization resulting in the appearance of higher condensed polyunsaturated graphite-like species containing oxygen groups [29,38]. A part of these functionalities can be stable under carbonization conditions $\left(850^{\circ} \mathrm{C}, 4 \mathrm{~h}\right)$. However, a majority of oxygen-containing groups appear during the exposure of the freshly carbonized sample to air.

\subsection{Catalytic activity of CMK-3 (2.00) replica in oxidative dehydrogenation of ethylbenzene}

Following the earlier reports, carbon materials have been considered to be active catalysts for the oxidative dehydrogenation (ODH) of ethylbenzene $[3,32,39,40]$,

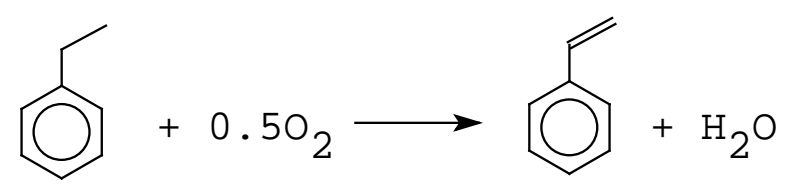

which is a promising alternative for the commercial method of styrene production. Activated carbons were extensively studied as the ODH catalysts [41-43]. Moreover, carbon nanomaterials (for instance: carbon nanofibers (CNFs), carbon nanotubes (CNTs), and onion-like carbons (OLCs)) were also tested in this role [44-48]. Use of carbon catalysts was proposed after discovery of so called 'active coke' mechanism of $\mathrm{ODH}[49,50]$. It was described that the formation of 'active coke' containing carbonyl/quinone groups on the surface of acidic materials (e.g. zeolites, alumina) in the initial period of the reaction resulted in an increase in their catalytic activity. 
The mesoporous CMK-3 (2.00) carbon replica synthesized using the PFA precursor seems an ideal ODH catalyst candidate. Its high surface area, size of mesopores as well as oxygen surface functionalities provide a high number of active sites, which are well accessible to the reactants $\mathrm{EB}$ and $\mathrm{O}_{2}$. The catalytic activity of the synthesized material in the process performed at an ethylbenzene/ $\mathrm{O}_{2}$ molar ratio of $1: 1$ has been studied. Styrene was the main reaction product within the studied temperature range, and only small amounts of $\mathrm{CO}_{\mathrm{x}}$, benzene and coke were formed as by-products. The EB conversion and yield of styrene vs. time-on-stream observed at different temperatures are shown in Supplementary Data (Fig. S4). It was found that the values of these parameters increase significantly with rising reaction temperature. It is especially noteworthy that no evident diffusion limitations were observed within the studied temperature range, which could be caused by carbon shell deposited on the outer surface. On the other hand, slight changes in catalytic activity of the studied sample are observed with time-on-stream. Obviously, the reaction system needs about $3 \div 5 \mathrm{~h}$ to achieve steady state. This effect, manifested by a decrease in the EB conversion and styrene yield, is opposite to the observations made for other acidic catalysts, which showed a gradual activation with time-on-stream attributed to the formation of active quinone sites [49,50].

After the initial reaction period, the catalytic activity of CMK-3 (2.00) is very stable. Table 4 presents the EB conversion, and yield/selectivity of products measured over this catalyst after $6 \mathrm{~h}$ time-on-stream. It is especially noteworthy that the studied replica is significantly more selective for styrene formation compared with the sucrose-derived replica, which was previously investigated by Su et. al. [51]. Nevertheless, it should be kept in mind that Su et. al. [51] used a higher content of oxidant, obtaining greater oxidation of $\mathrm{EB}$ to $\mathrm{CO}_{\mathrm{x}}$. Comparison of activity of the CMK-3 (2.00) replica with the catalysts tested previously under different reaction conditions is possible by calculation of $a$ parameter: 


$$
a=\frac{X_{E B} \cdot F_{E B, 0}}{W} \quad\left[\frac{\mu m o l}{g_{c a t} \cdot s}\right]
$$

where: $X_{E B}$ - conversion of EB expressed in molar fraction, $F_{E B, 0}-$ molar flow rate of EB in the inlet stream, $W-$ initial catalyst mass. At $350^{\circ} \mathrm{C}$ the $a$ value of 1.35 was achieved over CMK-3 (2.00). This value is only slightly lower than that measured for non-treated activated carbon (1.47) [41], and significantly higher compared with carbon nanotubes (0.80-1.12) [48].

The mesostructure of the synthesized replica is stable under the reaction conditions, as is confirmed by the isotherms of low-temperature $\mathrm{N}_{2}$ adsorption collected for the samples after the catalytic runs (Fig. S5). Nevertheless, after 6 h of catalytic test a considerable amount of carbonaceous deposit was formed on the CMK-3 surface. The coking yield depended strongly on the reaction temperature (cf. Table 4). The coke content increased in the spent catalysts tested up to $450^{\circ} \mathrm{C}$. When the catalytic run was performed at $500^{\circ} \mathrm{C}$ the rate of coke gasification surpassed the rate of coke deposition and $\Delta \mathrm{W}$ was relatively lower. Taking into account the textural parameters determined from low-temperature adsorption of $\mathrm{N}_{2}$ (Table $\mathrm{S} 2$ in Supplementary Data), it can be noticed that carbonaceous deposit blocked partially the channels in CMK-3. At low reaction temperatures micropores were clogged, whereas rising temperature to $400^{\circ} \mathrm{C}$ resulted also in plugging some mesopores.

Table 3 exhibits the changes in the composition of CMK-3 surface after the ODH process measured by XPS spectroscopy. It is especially noteworthy that during the catalytic performance in the presence of oxidizing agent at elevated temperatures, additional oxygen-containing functionalities appeared on the catalyst surface. Even after the catalytic run at the highest temperature the spent catalyst showed higher concentration of hydroquinone and quinine groups compared with the fresh sample. This observation can be evidence of catalytically active role of these species in the styrene production. 
Table 4 - Conversion of EB, yield and selectivity of main products and changes in catalyst mass measured in the ODH process after

$6 h$

\begin{tabular}{|c|c|c|c|c|c|c|c|c|c|c|}
\hline \multirow{2}{*}{$\begin{array}{c}\text { Reaction } \\
\text { temperature } \\
{\left[{ }^{\circ} \mathrm{C}\right]}\end{array}$} & \multirow{2}{*}{$\begin{array}{l}\text { Conversion } \\
\text { of EB } \\
\text { [\%] }\end{array}$} & \multicolumn{4}{|c|}{ Yield of products [\%] } & \multicolumn{4}{|c|}{ Selectivity of products [\%] } & \multirow{2}{*}{$\begin{array}{l}\Delta \mathrm{W}^{\mathrm{a}} \\
{[\%]}\end{array}$} \\
\hline & & Styrene & $\mathrm{CO}$ & $\mathrm{CO}_{2}$ & $\begin{array}{c}\text { Other } \\
\text { products }\end{array}$ & Styrene & $\mathrm{CO}$ & $\mathrm{CO}_{2}$ & $\begin{array}{l}\text { Other } \\
\text { products }\end{array}$ & \\
\hline 300 & 6.1 & 5.9 & 0.1 & 0.1 & 0.0 & 97.4 & 1.0 & 1.5 & 0.1 & 3.8 \\
\hline 350 & 17.4 & 16.7 & 0.3 & 0.4 & 0.0 & 95.9 & 1.5 & 2.4 & 0.2 & 9.4 \\
\hline 400 & 28.0 & 26.2 & 0.7 & 1.0 & 0.1 & 93.5 & 2.6 & 3.7 & 0.2 & 13.7 \\
\hline 450 & 38.2 & 34.6 & 1.5 & 1.9 & 0.2 & 90.5 & 4.1 & 4.9 & 0.5 & 15.8 \\
\hline 500 & 56.7 & 48.5 & 4.1 & 3.3 & 0.8 & 85.5 & 7.3 & 5.8 & 1.4 & 11.2 \\
\hline
\end{tabular}




\section{Conclusion}

A novel method of synthesis of CMK-3 carbon replica was developed. The carbon precursor, poly(furfuryl alcohol), was introduced into the SBA-15 silica template by the acid-catalyzed precipitation polycondensation technique. By adjusting a suitable mass ratio of monomer/SiO 2 in an aqueous slurry, the complete filling of the mesopore system was achieved. After hightemperature carbonization and removal of inorganic part of synthesized C/SBA-15 composite, the stable and well-ordered CMK-3 carbon replica was obtained. On account of the presence of oxygen-containing functional groups on the expanded carbon surface and its well-accessible pore system, the prepared material is an active catalyst for oxidative dehydrogenation of ethylbenzene to styrene.

\section{Acknowledgment}

This work was supported by the National Science Centre under the grant no. DEC2011/01/N/ST5/05595. Rafał Janus wishes to thank the Foundation for Polish Science MPD

Programme co-financed by the EU European Regional Development Fund for the financial support. The research was carried out with the equipment purchased thanks to the financial support of the European Regional Development Fund in the framework of the Polish Innovation Economy Operational Program (contract no. POIG.02.01.00-12-023/08).

\section{References}

[1] Ryoo R, Joo SH, Jun S. Synthesis of highly ordered carbon molecular sieves via templatemediated structural transformation. J Phys Chem B 1999;103:7745-7746. 
[2] Ryoo R, Joo SH. Nanostructured carbon materials synthesized from mesoporous silica crystals by replication. Stud Surf Sci Catal 2004;148:241-260.

[3] Figueiredo JL, Pereira MFR. The role of surface chemistry in catalysis with carbons. Catal Today 2010;150:2-7.

[4] Zhai Y, Wan Y, Cheng Y, Shi Y, Zhang F, Tu B, et al. The influence of carbon source on the wall structure of ordered mesoporous carbons. J Porous Mater 2008;15:601-611.

[5] Jun S, Joo SH, Ryoo R, Kruk M, Jaroniec M, Liu Z, Ohsuna T, Terasaki O. Synthesis of new, nanoporous carbon with hexagonally ordered mesostructure. J Am Chem Soc 2000;122:1071210713.

[6] Yokoi T, Seo S, Chino N, Shimojima A, Okubo T. Preparation of silica/carbon composites with uniform and well-ordered mesopores by esterification method. Microporous Mesoporous Mater 2009;124:123-130.

[7] Lei Z, An L, Dang L, Zhao M, Shi J, Bai S, et al. Highly dispersed platinum supported on nitrogen-containing ordered mesoporous carbon for methanol electrochemical oxidation. Microporous Mesoporous Mater 2009;119:30-38.

[8] Enterría M, Suárez-García F, Martínez-Alonso A, Tascón JMD. Synthesis of ordered micromesoporous carbons by activation of SBA-15 carbon replicas. Microporous Mesoporous Mater 2012;151:390-396.

[9] Lu A, Kiefer A, Schmidt W, Schüth F. Synthesis of Polyacrylonitrile-Based Ordered Mesoporous Carbon with Tunable Pore Structures. Chem Mater 2004;16:100-103.

[10] Fuertes AB. Synthesis of ordered nanoporous carbons of tunable mesopore size by templating SBA-15 silica materials. Microporous Mesoporous Mater 2004;67:273-281. 
[11] Fuertes AB, Nevskaia DM. Control of mesoporous structure of carbons synthesised using a mesostructured silica as template. Microporous Mesoporous Mater 2003;62:177-190.

[12] Fuertes AB, Nevskaia DM. Template synthesis of mesoporous carbons from mesostructured silica by vapor deposition polymerisation. J Mater Chem 2003;13:1843-1846.

[13] Fuertes AB. Template synthesis of mesoporous carbons with a controlled particle size. J Mater Chem 2003;13:3085-3088.

[14] Michorczyk P, Ogonowski J, Zeńczak K. Activity of chromium oxide deposited on different silica supports in the dehydrogenation of propane with $\mathrm{CO}_{2}$ - A comparative study. J Mol Catal A 2011;349:1-12.

[15] Lastoskie C, Gubbins KE, Quirke N. Pore Size Distribution Analysis of Microporous Carbons: A Density Functional Theory Approach. J Phys Chem 1993;97:4786-4796.

[16] Neimark AV, Lin Y, Ravikovitch PI, Thommes M. Quenched solid density functional theory and pore size analysis of micro-mesoporous carbons. Carbon 2009;47:1617-1628.

[17] Silvestre-Albero A, Silvestre-Albero J, Sepúlveda-Escribano A, Rodríguez-Reinoso F. Ethanol removal using activated carbon: Effect of porous structure and surface chemistry. Microporous Mesoporous Mater 2009;120:62-68.

[18] Joo SH, Ryoo R, Kruk M, Jaroniec M. Evidence for General Nature of Pore Interconnectivity in 2-Dimensional Hexagonal Mesoporous Silicas Prepared Using Block Copolymer Templates. J Phys Chem B 2002;106:4640-4646.

[19] Kruk M, Jaroniec M, Kim TW, Ryoo R. Synthesis and Characterization of Hexagonally Ordered Carbon Nanopipes. Chem Mater 2003;15:2815-2823.

[20] Lu AH, Li WC, Schmidt W, Kiefer W, Schüth F. Easy synthesis of an ordered mesoporous carbon with a hexagonally packed tubular structure. Carbon 2004;42:2939-2948. 
[21] Li Z, Yan W, Dai S. Surface Functionalization of Ordered Mesoporous Carbons - A Comparative Study. Langmuir 2005;21:11999-12006.

[22] Onfroy T, Guenneau F, Springuel-Huet MA, Gédéon A. First evidence of interconnected micro and mesopores in CMK-3 materials. Carbon 2009;47:2352-2357.

[23] Schmidt W. Calculation of XRD patterns of simulated FDU-15, CMK-5, and CMK-3 carbon structures. Microporous Mesoporous Mater 2009;117:372-379.

[24] Vinu A, Streb C, Murugesan V, Hartmann M. Adsorption of Cytochrome C on New Mesoporous Carbon Molecular Sieves. J Phys Chem B 2003;107:8297-8299.

[25] Lu AH, Schmidt W, Taguchi A, Spliethoff B, Tesche B, Schüth F. Taking Nanocasting One Step Further: Replicating CMK-3 as a Silica Material. Angew Chem Int Ed 2002;41:3489-3492.

[26] Huwe H, Fröba M. Synthesis and characterization of transition metal and metal oxide nanoparticles inside mesoporous carbon CMK-3. Carbon 2007;45:304-314.

[27] Gor GY, Thommes M, Cychosz KA, Neimark AV. Quenched solid density functional theory method for characterization of mesoporous carbons by nitrogen adsorption. Carbon 2012;50:1583-1590.

[28] Mariwala RK, Foley HC. Evolution of ultramicroporous adsorptive structure in poly(furfuryl alcohol)-derived carbogenic molecular sieves. Ind Eng Chem Res 1994;33:607-615.

[29] Burket CL, Rajagopalan R, Marencic AP, Dronvajjala K, Foley HC. Genesis of porosity in polyfurfuryl alcohol derived nanoporous carbon. Carbon 2006;44:2957-2963.

[30] Kruk M, Jaroniec M, Kim JM, Ryoo R. Characterization of highly ordered MCM-41 silicas using X-ray diffraction and nitrogen adsorption. Langmuir 1999;15:5279-5284.

[31] Kruk M, Jaroniec M, Sayari A. Adsorption study of surface and structural properties of MCM-41 materials of different pore sizes. J Phys Chem B 1997;101:583-589. 
[32] Serp P, Figueiredo JL. Carbon Materials for Catalysis. New Jersey: John Wiley \& Sons; 2009.

[33] Rodríguez-Reinoso F, Molina-Sabio M. Textural and chemical characterization of microporous carbons. Adv Colloid Interf 1998;76-77:271-294.

[34] Darmstadt H, Roy C, Kaliaguine S, Choi SJ, Ryoo R. Surface chemistry of ordered mesoporous carbons. Carbon 2002;40:2673-2683.

[35] Zielke U, Hüttinger KJ, Hoffman WP. Surface-oxidized carbon fibers: I. Surface structure and chemistry. Carbon 1996;34:983-998.

[36] Takahagi T, Ishitani A. XPS study on the surface structure of carbon fibers using chemical modification and C1s line shape analysis. Carbon 1988;26:389-395.

[37] Weidenthaler C, Lu AH, Schmidt W, Schüth F. X-ray photoelectron spectroscopic studies of PAN-based ordered mesoporous carbons (OMC). Microporous Mesoporous Mater 2006;88:238243.

[38] Janus R, Wach A, Kuśtrowski P, Dudek B, Drozdek M, Silvestre-Albero AM, et al. Investigation on the low-temperature transformations of poly(furfuryl alcohol) deposited on MCM-41. Langmuir 2013;29:3045-3053.

[39] Grunewald GC, Drago RS. Oxidative dehydrogenation of ethylbenzene to styrene over carbon-based catalysts. J Mol Catal 1990;58:227-233.

[40] Guerrero-Ruiz A, Rodríguez-Ramos I. Oxydehydrogenation of ethylbenzene to styrene catalyzed by graphites and activated carbons. Carbon 1994;32:23-29.

[41] Pereira MFR, Órfão JJM, Figueiredo JL. Oxidative dehydrogenation of ethylbenzene on activated carbon catalysts. I. Influence of surface chemical groups. Appl Catal A 1999;184:153160. 
[42] Pereira MFR, Órfão JJM, Figueiredo JL. Oxidative dehydrogenation of ethylbenzene on activated carbon catalysts 2. Kinetic modelling. Appl Catal A 2000;196:43-54.

[43] Pereira MFR, Órfão JJM, Figueiredo JL. Oxidative dehydrogenation of ethylbenzene on activated carbon catalysts 3. Catalyst deactivation. Appl Catal A 2001;218:307-318.

[44] Delgado JJ, Chen XW, Frank B, Su DS, Schlögl R. Activation processes of highly ordered carbon nanofibers in the oxidative dehydrogenation of ethylbenzene. Catal Today 2012;186:9398.

[45] Mestl G, Maksimowa NI, Keller N, Roddatis VV, Schlögl R. Carbon nanofilaments in heterogeneous catalysis: an industrial application for new carbon materials? Angew Chem Int Ed 2001;40:2066-2068.

[46] Delgado JJ, Vieira R, Rebmann G, Su DS, Keller N, Ledoux MJ, et al. Supported carbon nanofibers for the fixed-bed synthesis of styrene. Carbon 2006;44:809-812.

[47] Guerrero-Ruiz A, Rodríguez-Ramos I. Oxydehydrogenation of ethylbenzene to styrene catalyzed by graphites and activated carbon. Carbon 1994;32:23-29.

[48] Pereira MFR, Figueiredo JL, Órfão JJM, Serp P, Kalck P, Kihn Y. Catalytic activity of carbon nanotubes in the oxidative dehydrogenation of ethylbenzene. Carbon 2004;42:2807-2813. [49] Emig G, Hofmann H. Action of zirconium phosphate as a catalyst for the oxydehydrogenation of ethylbenzene to styrene. J Catal 1983;84:15-26.

[50] Cavani F, Trifirò F. Alternative processes for the production of styrene. Appl Catal A 1995;133:219-239.

[51] Su DS, Delgado JJ, Liu X, Wang D, Schlögl R, Wang L, et al. Highly ordered mesoporous carbon as catalyst for oxidative dehydrogenation of ethylbenzene to styrene. Chem Asian J 2009;4:1108-1113. 